\title{
Underlying Mechanisms Between Diabetes Mellitus and Osteoporosis
}

\author{
Oscar Rosero Olarte ${ }^{1}$ and Mauricio Alvarez Andrade ${ }^{2}$ \\ 1. Instituto de Osteoporosis de los Llanos, Osteollanos, Regional Hospital, Villavicencio, Colombia; 2. Central Military Hospital, Bogotá, Colombia
}

DOI: https://doi.org/10.17925/USE.2018.14.2.65

S everal studies have linked diabetes mellitus with an increased risk of bone fractures. Patients with type 1 diabetes have lower bone mineral density and patients with type 2 diabetes have an increased risk of fracture despite normal or high bone mineral density, measured by dual energy x-ray absorptiometry. Different mechanisms have been proposed in the pathophysiology of bone disease in diabetic patients. The mechanisms involved include microvasculature changes, advanced glycation end products accumulation, and altered osteoblast-osteoclast function with increased sclerostin, decreased insulin-like growth factor 1 (IGF1), decreased osteocalcin, and other molecular alterations, leading to a decrease in bone turnover and altered bone quality. Other factors, such as increased risk of falls related to diabetic neuropathy and diabetic retinopathy have also been proposed. Different diagnostic methods have been described for the study of bone disease in patients with diabetes, due to the lack of correlation between bone mineral density measured by dual $\mathrm{x}$-ray absorptiometry and the fracture risk in this group of patients. These methods include the evaluation of bone turnover markers, the FRAX score, the trabecular bone score, and the evaluation of microarchitecture with high-resolution peripheral quantitative tomography. This editorial provides a short overview of the underlying mechanisms between diabetes mellitus and osteoporosis, and the diagnostic methods used to evaluate these patients.

\section{Keywords}

Diabetes mellitus, osteoporosis,

bone tissue, bone density

Disclosure: Oscar Rosero Olarte and Mauricio Alvarez Andrade have nothing to disclose in relation to this article.

Review Process: This article is a short opinion piece and has not been submitted to external peer reviewers, but was reviewed by the Editorial Board before publication.

Authorship: All named authors meet the International Committee of Medical Journal Editors (ICMJE) criteria for authorship of this manuscript, take responsibility for the integrity of the work as a whole, and have given final approval to the version to be published.

open Access: This article is published under the Creative Commons Attribution Noncommercial License which permits any noncommercial use, distribution, adaptation, and reproduction provided the original author(s) and source are given appropriate credit.

Received: June 1, 2018

Accepted: September 19, 2018

Citation: US Endocrinology, 2018;14(2):65-6

Corresponding Author: Oscar Rosero Olarte, Instituto de Osteoporosis de los Llanos, Cra. 44b \#33b114, Villavicencio, Meta, Colombia 500001. E: oscarroseromd@yahoo.com

Support: No funding was received in

the publication of this article.
Diabetes mellitus has been associated with decreased bone quality and higher risk of fractures in several studies. ${ }^{1-3}$ Patients with type 1 diabetes have lower bone mineral density and patients with type 2 diabetes have an increased risk of fracture despite normal or high bone mineral density, measured by dual energy $x$-ray absorptiometry. ${ }^{1,45}$ While some studies have shown an increased risk of vertebral fracture in patients with diabetes, others have found the same risk in patients with and without diabetes. ${ }^{1-3,6}$

The exact mechanism of bone disease in diabetes is not clear. ${ }^{7-9}$ Several studies have found very complex mechanisms that range from the cellular level, with molecular alterations in cell signaling that alter the bone turnover, to changes in the microarchitecture and microvascular quality of bone..$^{8-11}$ Other factors that contribute to the increased fracture risk include complications of diabetes, such as diabetic retinopathy, diabetic neuropathy, increased risk of falls, and antidiabetic drugs. ${ }^{10,11}$

One of the main factors involved in the pathophysiology of bone disease in patients with type 1 diabetes is the absolute absence of insulin. Insulin has an anabolic effect on the bone; therefore, the absence of insulin results in lower bone formation rates and lower peak bone mass during growth. ${ }^{11}$ A recent systematic review found that lower insulin-like growth factor 1 (IGF1) levels in patients with type 1 diabetes seem to be responsible for the decrease in bone turnover. ${ }^{10}$ However, bone turnover markers do not seem to differ from that of control patients, with markers such as 25-hydroxyvitamin D, carboxyterminal propeptide type I, procollagen, type I collagen, and cross linked carboxy-terminal telopeptide. In type 1 diabetes evidence is conflicting, but it would seem that low bone turnover is a multifactorial and may be related to other factors like diabetes stage, diabetes complications, age, medications and gender. ${ }^{10}$

Reni at al. they found that, in both in vivo and in vitro experiments, there is increased osteoclast activation trigged by hypoxia and metabolic acidosis, and mediated by reduced receptor activator of nuclear factor kappa-B ligand (RANKL) levels and an increase in osteoprotegerin (OPG), leading to a reduction in the RANKL/OPG ratio. ${ }^{12}$ This study concluded metabolic acidosis is an important factor that triggers osteoclast activation in type 1 diabetic patients.

In type 2 diabetes, there are a lot of factors that increase the risk of fractures. These factors include sarcopenia and poor balance, as well as diabetic complications such as diabetic 
neuropathy, diabetic retinopathy, hypoglycemia, and diabetes drugs.11 At the molecular level, advanced glycation end products (AGES), like pentosidine, produced from increase glucose exposure in tissues, seems to be an important mechanism. In vitro studies have shown that AGEs suppress mineralization by inducing transforming growth factor-beta (TGF- $\beta$ ) activity, inhibition of osteoblast differentiation, and mineralization of osteoblastic cells?

A decrease in bone formation has been proposed as an additional factor for bone disease in type 2 diabetes. ${ }^{11}$ Lower levels of bone formation markers, such as the amino-terminal propeptide of type I collagen and osteocalcin have been found in diabetic patients, as well as markers of bone reabsorption such as cross linked carboxy-terminal telopeptide of type I collagen. ${ }^{11}$ Sclerostin, a WNT signaling pathway inhibitor, is higher in diabetic patients and seems to be responsible for low bone formation in this group of patients. ${ }^{10}$ This produces a state of low bone turnover that results in bone microdamage, altering bone quality. The reason for the lower bone resorption is not well understood, but seems to be responsible for the bone mineral density alterations found in patients with type 2 diabetes. ${ }^{11}$ Decreases in IGF1 and osteocalcin seem to be factors that, when associated with high levels of sclerostin, contribute to the reduction of bone turnover in type 2 diabetic patients. ${ }^{10}$

Diabetes is an independent risk factor for osteoporosis related fractures. Risk fracture assessment tools, such as FRAX, underestimate fracture risk in diabetic patients. ${ }^{13}$ Other tools, such as the trabecular bone score, have shown to improve discrimination of patients with poor bone quality despite normal bone mineral density measured by dual energy $\mathrm{x}$-ray absorptiometry. ${ }^{14}$ Two studies used high-resolution computed tomography imaging to evaluate bone quality. ${ }^{15,16}$ They found that patients with type 2 diabetes have higher trabecular bone mineral density, with increased cortical porosity. This is probably due to microvascular disease in the bone. These changes can produce alterations in bone mineral density values measured by standard techniques, despite an increased risk in bone fracture.

Available evidence shows a clear association between diabetes and impaired bone quality that predisposes to fractures. More studies are needed to clarify the most important mechanisms in the pathophysiology of bone quality deterioration in these patients and to determine the best option for the diagnosis of bone fragility in diabetic patients.
1. Vestergaard P. Discrepancies in bone mineral density and fracture risk in patients with type 1 and type 2 diabetes-a meta-analysis. Osteoporos Int. 2007:18:427-44.

2. Napoli N, Strotmeyer ES, Ensrud KE, et al. Fracture risk in diabetic elderly men: the MrOS study. Diabetologia. 2014;57:2057-65.

3. Janghorbani M, Van Dam RM, Willett WC, Hu FB. Systematic review of type 1 and type 2 diabetes mellitus and risk of fracture. Am J Epidemiol. 2007;166:495-505.

4. Bonds DE, Larson JC, Schwartz AV, et al. Risk of fracture among women with type 2 diabetes: the women's health initiative observational study. J Clin Endocrinol Metab. 2006;91:3404-10.

5. Rubin MR. Skeletal fragility in diabetes. Ann NY Acad SCi. 2017:1402:18-30.

6. Napoli N, Schwartz AV, Schafer AL, et al. Osteoporotic fractures in men (MrOS) Study Research Group. J Bone Miner Res. 2018;33:63-9.
7. Masakazu N, Toru Y, Kyoto O, et al. Advanced glycation end product 3 (AGE3) suppresses the mineralization of mouse stroma ST2 cells and human mesenchymal stem cells by increasing TGF- $\beta$ expression and secretion. Endocrinology. 2014:155:2402-10

8. Merlotti D, Gennari L, Dotta F, et al. Mechanisms of impaired bone strength in type 1 and 2 diabetes. Nutrition, Metabolism \& Cardiovascular Diseases. 2010;20:683-90

9. Roy B. Biomolecular basis of the role of diabetes mellitus in osteoporosis and bone fractures. World I Diabetes. 2013;4:101-13.

10. Starup-Linde J, Vestergaard P. Biochemical bone turnover markers in diabetes mellitus - A systematic review. Bone. 2016;82:69-78.

11. Farr JN, Khosla S. Determinants of bone strength and quality in diabetes mellitus in humans. Bone. 2016:82:28-34.

12. Reni $C$, Mangialardi G, Meloni M, Madeddu P. Diabetes stimulates osteoclastogenesis by acidosis-induced activation of transient receptor potential cation channels. Sci Rep. 2016;6:30639

13. Giangregorio LM, Leslie WD, Lix LM, et al. FRAX underestimates fracture risk in patients with diabetes. $J$ Bone Miner Res. 2012:27:301-8.

14. KL, De Abreu LLF, Hans D, et al. Trabecular bone score in men and women with impaired fasting glucose and diabetes. Calcif Tissue Int. 2018;102:32-40.

15. Burghardt AJ, ISsever AS, Schwartz AV, et al. High-resolution peripheral quantitative computed tomographic imaging of cortical and trabecular bone microarchitecture in patients with type 2 diabetes mellitus. J Clin Endocrinol Metab. 2010:95:5045-55

16. Shanbhogue $\mathrm{W}$, Hansen $\mathrm{S}$, Frost $\mathrm{M}$, et al. Compromised cortica bone compartment in type 2 diabetes mellitus patients with microvascular disease. Eur J Endocrinol. 2016;174:115-24. 